The Journal of SPORT, 2014, 3(1), 63-101

(C) Kent State University

\title{
Achievement Goal Orientation as a Predictor of Sport Involvement and Perceived Benefits of Sport Involvement: Examination of a Mixture Model
}

\author{
Leeann M. Lower \\ The Ohio State University \\ Brian A. Turner \\ The Ohio State University \\ Jeffrey C. Petersen \\ Baylor University
}

\begin{abstract}
Grounded in the dichotomous achievement goal framework, this study examined the utility of achievement goal orientations to predict sport involvement and perceived benefits (social, intellectual, and fitness) associated with participation in three college recreational sport programs: group fitness, intramural sport, and sport clubs. A questionnaire, based on the Task and Ego Orientation in Sport Questionnaire (TEOSQ) and Quality and Importance of Recreational Services (QIRS) perceived benefit scale, was administered to recreational sport participants $(n=1,564)$ at a single institution. A mixture model was proposed and tested, for which task orientation was found to positively predict sport involvement and perceived benefits of involvement, while ego orientation only predicted sport involvement. Sport involvement was found to positively predict perceived benefits of involvement. Implications for sport practitioners include task goal orientation enhancement within sport offerings and increased involvement opportunities, while theoretical implications can guide future achievement goal research within the sport domain.
\end{abstract}


Sport is a type of achievement activity that has received considerable attention in management and psychology literature. Theoretical and empirical investigation of the achievement goal construct emerged from a desire to account for achievement behavior and help guide individuals to adopt optimal motivation in achievement settings (Elliot, 2005). The achievement goal construct is considered among the strongest predictors of achievement-related behavior and outcomes (Elliot \& McGregor, 2001; Moller \& Elliot, 2005). Research examining the predictive relationship between achievement goal orientations, behaviors, and outcomes can guide effective program structuring and interventions facilitated by sport practitioners to influence participant dispositions in order to produce positive behaviors and outcomes.

The purpose of this study was to examine a structural model incorporating achievement goal orientations, recreational sport involvement (i.e., achievement behavior), and perceived benefits of recreational sport involvement (i.e., achievement outcome). Such assessment can advance achievement goal theory and involvement theory by gaining a better understanding of the antecedents and consequences of achievement goal orientations and sport involvement. In addition to furthering the body of knowledge, the findings of this study can be used to shed light on optimal dispositions within sport which can help practitioners produce improved participant outcomes through sport programs.

\section{Recreational Sport}

\section{Review of Literature}

Recreational sport is one of many conduits of extracurricular activities available on most American college campuses. The recreational sport programs that the current study examined include group fitness, intramural sports, and sport clubs. Group fitness programs typically operate in a system of classes through which trained student instructors facilitate physical exercises. The dynamic structure of group fitness enables the program to meet the interests of a diverse population, emphasizing effort and personal achievement. Intramural sports emerged through student initiated 
athletic competitions, prior to the establishment of varsity athletics on college campuses (Bourgeois et al., 1995; Staurowsky \& Abney, 2011). The purpose of intramural sport is to provide an avenue for a wider cross-section of students to participate in competitive games with students enrolled at the same university. "The role of sports club programs has changed from providing the foundation and development for varsity athletic programs to becoming an important recreation program alternative" (Cooney, 1979, p. 40). Sport clubs are designed to be an opportunity for students to participate in competitive sport outside the confines of their own institution, in which club members organize and host the competitions.

Involvement with recreational sport on college campuses has been associated with learning outcomes, student development, and additional beneficial outcomes (NIRSA/NASPA Consortium, 2010; Haines \& Fortman, 2008; The Ohio State University, 2003). While recreational sport assessment often focuses on positive outcomes, achievement goal literature considers both positive and negative outcomes, such as persistence, effectiveness, and anxiety (Elliot, 2005; Hendricks \& Payne, 2007; Roberts, Treasure, \& Balague, 1998). Perceived benefits of sport participation were selected as the achievement-related outcome measure due to the secondary purpose of this study which aimed at examining the value of sport programs.

\section{Perceived Benefits of Involvement}

In an effort to understand the potential gains of sport programs, investigators have developed instruments for the purpose of measuring outcomes of program participation, such as perceived benefits. The literature emphasizes three benefit subgroups associated with physical activity programs: intellectual, social, and fitness benefits (Artinger et al., 2006; Haines, \& Fortman, 2008; NIRSA, 2004). Perceived benefits are typically assessed through scales measuring one's perception of gains realized through participation. Several instruments have been developed to assess perceived benefits of recreational sport involvement, including: Exercise Benefits/Barriers Scale (EBBS), Measuring Outcomes from 
Recsports Experiences (MORE), and Quality and Importance of Recreational Services (QIRS) perceived benefit scale.

The QIRS survey, developed by the National IntramuralRecreational Sport Association (NIRSA), is of primary importance to this study; its saliency within recreational sport literature has been demonstrated (NIRSA, 2000). The perceived benefit scale is a component within the QIRS survey which focuses on assessing perceived benefits associated with recreational sport involvement. The perceived benefits scale was selected for the purpose of this study based upon its focus on the dominant benefit subgroups and brevity.

\section{Involvement}

Within sport literature, involvement is typically synonymous with participation. However there are varying types of participation that must be considered (e.g., coach, player, spectator, employee) when deciphering involvement. Theoretical frameworks have been developed to offer foundational perspectives from which to interpret the construct. Within Astin's theory (1999), involvement refers to the time and energy spent participating in an academic or extracurricular experience, incorporating both a quantitative and qualitative component (Astin, 1999; Kuh, 1991). Quantitative involvement pertains to the amount of time spent participating in an activity while qualitative involvement emphasizes degree of effort or energy expended. Within the literature, quantitative involvement is more frequently utilized than qualitative involvement likely due to its objective nature which allows for ease of measurement.

For the purpose of this study, involvement focused on quantity of time spent participating as opposed to qualitative involvement. Involvement was selected as a measure of achievement-related behavior to facilitate the investigation of the hypothesized relationship between the achievement goal construct and achievement-related behavior. The construct was also selected based upon empirical research which has found involvement associated with cognitive, affective, and overall student development, which closely aligns with the perceived benefit groups 
of interest within this current study (Astin, 1999; Hall, 2006; Lindsey \& Sessoms, 2006).

\section{Achievement Goal Theory}

The achievement goal construct emerged in the 1970s through the independent and collaborative efforts of Ames, Dweck, Maehr, and Nicholls (Roberts, 2001; Elliot, 2005). Achievement goal theory stems from a social cognitive perspective of attribution (LeUnes, 2008). Attribution theory is a prominent motivational model that examines the underlying causes of an individual's behavior. Motivation can be defined by the constructs that "energize, direct, and regulate achievement behavior", where achievement refers to the attainment of an achievement goal (Roberts, Treasure, \& Conroy, 2007, p. 3). Achievement goal theory assumes that achievement goals guide (i.e., motivate) achievement-related behavior and reflect anticipated outcomes of this behavior (Roberts et al., 2007). Achievement goals often constitute the purpose of task engagement (Elliot, 1999). A social cognitive approach to motivation assumes that individuals actively engage in making decisions regarding their achievement behavior, which reflects the achievement goal construct (Roberts, 2001; Roberts et al., 2007). Achievement itself is a construct subjectively defined; success or failure of attaining an achievement goal is based upon an individual's subjective assessment of the outcome.

Competency and valence are two fundamental elements within the achievement goal theory. Nicholls (1984) proposed that two primary conceptions of ability manifest in achievement contexts - undifferentiated and differentiated concepts of ability, which delineate how competency is defined. The two conceptions of ability represent different criteria for assessing one's ability. An undifferentiated concept of ability associates ability with effort, therefore the more effort one expends, the greater learning (indication of ability) one achieves - linking effort and perceived ability. While the undifferentiated approach associates ability and effort, the differentiated concept of ability differentiates between ability and effort, for which ability is perceived as capacity. By 
differentiating ability and effort, one might learn through effort yet fail to demonstrate high ability. The two conceptions of ability constitute the source of criteria used to assess success and failure (Roberts et al., 2007). Three standards have been utilized in competence evaluation - absolute, intrapersonal, and normative. An undifferentiated concept of ability assesses ability through personal improvement (intrapersonal evaluation) or mastery (absolute evaluation). A differentiated concept of ability assesses ability through interpersonal comparison of effort and ability, for which success is associated with outperforming others (normative evaluation). Nicholls labeled the adoption of an undifferentiated concept of ability as task involvement and the adoption of a differentiated concept of ability as ego involvement. Ames and Archer (1988) identified terminology used synonymously with task and ego goals within achievement goal literature (e.g., task: learning, mastery; ego: performance, ability) and recommended the terms 'mastery' and 'performance' goals; however, some researchers have continued to use Nicholls' original task-ego labels (Elliot, 2005). For the purpose of this study, achievement goals will be labeled 'task' and 'ego'.

Achievement goal theorists have hypothesized an association between task goals and positive, adaptive processes and outcomes (e.g., enhanced task enjoyment, effort in the face of difficulty) and between ego goals and negative, maladaptive processes and outcomes (e.g., reduced persistence in the face of difficulty, avoiding challenge; Elliot, 1999, 2005; Roberts et al., 1998). When considering achievement goals characterized by how competency is defined (i.e., task and ego goals), research has demonstrated relatively consistent findings regarding the consequences of task goals (positive), yet inconsistent findings regarding the consequences of ego goals (positive, neutral, negative; Elliot, 2005; Roberts, Treasure, \& Kavussanu, 1996). The inconsistent results fail to support the original hypothesized relationship between ego goals and negative processes and outcomes. Researchers have considered the capacity of task goals to moderate the potentially negative effects of ego goals to explain the inconsistencies in the literature (Roberts 
et al., 1996). Other researchers have incorporated an approachavoidance motivation distinction to help explain the varied results (Elliot, 1999).

Approach and avoidance motivation are considered a function of valence. Competence is valenced in that it is conceptualized as either a positive possibility (i.e., competency, success) or negative possibility (i.e., incompetency, failure); these conceptions have been linked with approach and avoidance tendencies (Elliot \& Covington, 2001; Lang, 1995). The approachavoidance distinction was incorporated in the first formal model of achievement motivation (Lewin, Dembo, Festinger, \& Sears, 1944). With the emergence of the achievement goal construct in the 1970s and $80 \mathrm{~s}$, the approach-avoidance distinction was overlooked. Dweck and Nicholls maintained a contemporary conceptualizing of achievement goals, focusing on how competency is defined. Dweck considered achievement goals to be omnibus constructs that included a combination of approach-avoidance tendencies, while Nicholls characterized both achievement goals (task and ego) as approachoriented (Elliot, 1999). In the 1990s, Elliot and colleagues returned to the incorporation of the approach-avoidance distinction to address inconsistent findings associated with use of the dichotomous conceptual framework and extend achievement goal theory (Adie, Duda, \& Ntoumanis, 2008; Elliot, 1999; Roberts et al., 2007).

\section{Achievement Goal Models}

Dichotomous model. The contemporary achievement goal approach is conceptualized as dichotomous or orthogonal. Dweck and Nicholls are primarily recognized for the development and utilization of this dichotomous approach (Dweck \& Leggett, 1988; Nicholls, 1989). Dweck and Leggett (1988) conducted their theory of intelligence, involving achievement goals, as a dichotomous variable, regarding the construct as omnibus with both approach and avoidance tendencies. Although Nicholls (1984) defined achievement behavior as demonstrating high ability or avoiding demonstrating low ability, he later proposed orthogonal goal orientations characterized as approach-oriented (Duda, 2005; Elliot, 
2005; Roberts et al., 1996). The orthogonal viewpoint perceives goal orientations as independent, in which one can possibly have both dispositions at the same time (Roberts et al., 1996). The orthogonal interpretation of achievement goals has been empirically supported (Roberts et al., 1996); however, with the construct having theoretically evolved towards the inclusion of the approachavoidance distinction, the conceptualization has followed the dichotomous (i.e., divided) structure.

The dichotomous achievement goal approach was selected for the purpose of this study based upon the following rationale. The task-ego (i.e., mastery-performance) structure is recognized as having simple and straightforward features and has gained widespread attention in the literature (Elliot \& McGregor, 2001). The approach-avoidance distinction has yielded inconsistent results. Past research has collapsed approach-avoidance motivation suggesting similarities between the constructs, while more recent research supports the distinction (Duda, 2005; Elliot, Murayama, \& Pekrun, 2011). Research investigating the predictive utility of the $2 \times 2$ framework was suggested to be parsimonious in 2008 (Adie et al., 2008), and the 3x2 approach was just introduced in 2011 (Elliot, Murayama, \& Pekrun, 2011). Overall, the dichotomous approach was selected because of its simplistic, adaptable nature and widespread usage, as well as due to the changing developments in regards to the valence dimension.

Alternative models. Elliot and Harackiewicz (1996) expanded the dichotomous framework by incorporating the approach-avoidance distinction within the performance (i.e., 'ego') goal, resulting in a trichotomous framework. Later, Elliot concluded that the trichotomous framework was designed to only consider positive mastery possibilities (associated with an approach orientation) (Elliot, 2005), therefore Elliot and McGregor (2001) expanded the trichotomous framework by incorporating the approach-avoidance distinction within the mastery (i.e., 'task') goal, creating a $2 \times 2$ framework. The $2 \times 2$ framework was expanded to the most recent achievement goal approach $(3 \times 2)$ by Elliot, Murayama, and Pekrun (2011). Elliot and colleagues suggest the division of 
task-based and self-based goals based upon the possibility of pursuing these goals independently. Within the $3 \times 2$ framework, the authors define competency by three types of achievement goals (task, self, other), while maintaining the approach-avoidance distinction.

\section{Goal Orientation}

A state of goal involvement (task or ego) depends on one's dispositional goal orientation and situational factors (e.g., perceived motivational climate; Roberts, 2001). Goal orientation reflects an individual's predisposition towards task or ego goal involvement (Roberts et al., 2007). Goal orientation is not a personal trait, rather a cognitive schema that may be subject to change, such as through socialization. Scholars have demonstrated the relative stability of goal orientations over time but suggest a malleable quality in that goal orientations may be impacted by situational factors (Ames, 1992; Duda \& Whitehead, 1998; Sage, \& Kavussanu, 2008).

To measure an individual's predisposition in achievement contexts, researchers have developed questionnaires incorporating criteria one might associate with success (i.e., demonstrating competence, avoiding the demonstration of incompetence). The questionnaires assess one's perception of the evaluation criteria in order to estimate one's disposition. The Task and Ego Orientations in Sport Questionnaire (TEOSQ; Duda, 1989; Duda, 1998; Roberts et al.,1998) was selected for the purpose of this study based upon its prominence throughout goal orientation literature and dichotomous framework (Biddle, Want, Kavussanu, \& Spray, 2003). A state of goal involvement (task or ego) depends on one's dispositional goal orientation and situational factors (e.g., perceived motivational climate; Roberts, 2001). Goal orientation reflects an individual's predisposition towards task or ego goal involvement (Roberts et al., 2007). Goal orientation is not a personal trait, rather a cognitive schema that may be subject to change, such as through socialization.

Scholars have demonstrated the relative stability of goal orientations over time but suggest a malleable quality in that goal orientations 
may be impacted by situational factors (Ames, 1992; Duda \& Whitehead, 1998; Sage, \& Kavussanu, 2008).

To measure an individual's predisposition in achievement contexts, researchers have developed questionnaires incorporating criteria one might associate with success (i.e., demonstrating competence, avoiding the demonstration of incompetence). The questionnaires assess one's perception of the evaluation criteria in order to estimate one's disposition. The Perception of Success Questionnaire and Task and Ego Orientations in Sport Questionnaire (TEOSQ) are prominent instruments that adopt the dichotomous framework with an orthogonal perspective.

The TEOSQ emerged from the Achievement Motivation Scale (i.e., Motivational Orientation Scale) developed by Nicholls and colleagues (Nicholls, Patashnick, \& Nolen, 1985). Nicholls and Duda modified the Achievement Motivation Scale for the sport domain and utilized conceptual definitions of achievement goal orientations for the development of the TEOSQ (Duda, 1989; Duda, 1998; Roberts et al.,1998). The TEOSQ has been used in a multitude of studies to measure dispositional goal orientations (Biddle, Want, Kavussanu, \& Spray, 2003).

A systematic review of research by Biddle et al., (2003), investigated the correlates of dispositional goal orientations. The review was limited to studies that used either the TEOSQ or the POSQ in the sport and physical activity domains and were published between 1990 and 2000. The systematic review analyzed 98 studies of which $80.6 \%$ used the TEOSQ to measure dispositional goal orientations. Based upon the prominence of the instrument throughout goal orientation literature, this study employed the TEOSQ to measure dispositional goal orientations.

\section{Integration of Constructs}

The achievement goal theory stems from a social cognitive perspective of attribution (LeUnes, 2008). Social cognitive theory suggests that personal factors, environmental factors, and behavior are reciprocal determinants of each other (Dishman \& Chambliss, 2010). Within this study, the personal factor examined was goal 
orientation while behavior was represented by degree of recreational sport involvement. Achievement goal literature considers the achievement goal construct one of the strongest predictors of achievement-related behavior and outcomes (Elliot \& McGregor, 2001; Moller \& Elliot, 2006). For the purpose of this study, perceived benefits of recreational sport participation represented achievement-related outcomes. The link between achievement goals and achievement-related behavior and outcomes is motivation - the underlying causes of an individual's behavior and expected outcomes of that behavior.

Past achievement goal theorists have suggested task goals to be associated with positive, adaptive processes and outcomes and ego goals to be associated with negative, maladaptive processes and outcomes (Elliot, 1999, 2005; Roberts et al., 1998). A task orientation has received greater support because evaluation of one's success is within the individual's control. Literature has often suggested the depression of an ego orientation based upon past empirical studies which have linked ego orientation with negative behaviors and outcomes. An ego oriented individual is not able to control the likelihood of success because he/she cannot control the performance of others, which is why maladaptive achievement behaviors are often exhibited when challenges arise (Roberts et al., 1998).Empirical studies have demonstrated relatively consistent findings regarding the consequences of task goals (positive), yet inconsistent findings regarding the consequences of ego goals (positive, neutral, negative; Elliot, 2005; Roberts et al., 1996). Roberts et al., (1996) suggest that the potential negative behaviors and outcomes associated with an ego orientation can be moderated by enhancing an individual's task orientation. Further investigation is needed to confirm the type of consequences associated with a task goal orientation and illuminate the inconsistency with the ego goal orientation construct. In regards to the relationship between achievement goal orientations and achievement-related behaviors and outcomes, it was hypothesized that achievement goals will directly predict sport involvement and indirectly predict perceived benefits of sport involvement. 
Astin (1999) postulates a positive direct relationship between involvement and student development; empirical research has also found involvement associated with cognitive, affective, and overall student development. The student development outcome closely aligns with the perceived benefit outcomes investigated within this current study (Astin, 1999; Hall, 2006; Lindsey \& Sessoms, 2006), therefore it was hypothesized that sport involvement will positively predict perceived benefits of sport involvement. A structural model (Figure 1) integrating the achievement goal orientation (i.e., task and ego), recreational sport involvement, and perceived benefits of recreational sport variables was proposed and tested.

Figure 1

Path diagram for proposed structural model

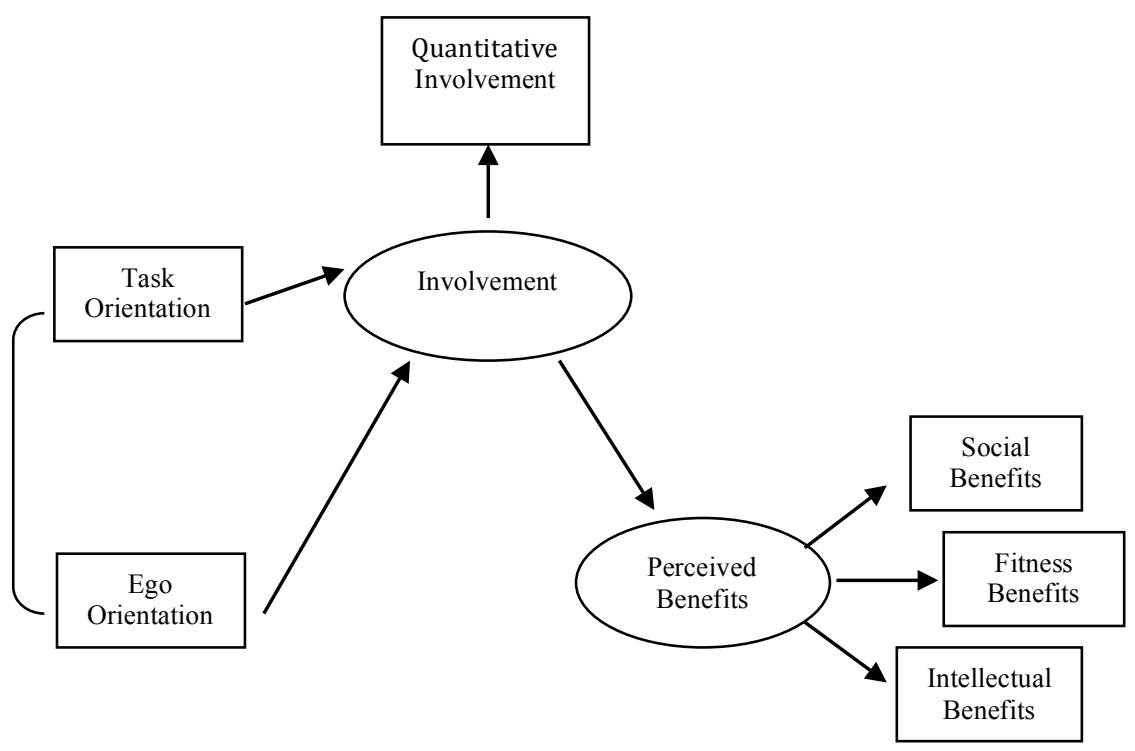




\section{Participants}

\section{Methods}

The study was conducted at a mid-sized, post-secondary institution in the Southwestern United States. The target population included participants involved in one of three recreational sport programs on campus: group fitness, intramural sports, and sport clubs. The sampling frame consisted of 6,467 subjects. A nonprobability, convenience sampling technique was utilized. A total of 1,881 subjects participated in the study; after eliminating duplicate questionnaires, incomplete cases, and cases constituted as outliers (based upon recreational sport involvement), the sample size comprised 1,564 subjects.

\section{Instrumentation}

The research instrument included a total of 44 items organized within three primary sections: demographics (6 items), a modified version of the TEOSQ (12 items), and a modified version of the QIRS survey (22 items). The demographic section included data regarding: recreational sport involvement, gender, age, academic year, and ethnic group. Quantitative involvement in recreational sport was measured by minutes of participation per week for each of the three recreational sport program areas (i.e., group fitness, intramural sports, and sport clubs).

The TEOSQ was used to determine the achievement goal orientation of the subjects. The instrument was designed to assess an individual's proneness for task and ego involvement (Duda, 1998). The TEOSQ uses a 5-point Likert scale ranging from 1 (strongly disagree) to 5 (strongly agree). For the purpose of this study, the questionnaire was modified to 12 items (six ego orientation items, six task orientation items). The modification allowed for each domain to be represented equally.

Validity and reliability of the TEOSQ instrument was established by Duda (1998). Reliability was established by test-retest and internal consistency. Test-retest resulted in an $r=.68$ and $r=.75$ after a three week period respectively, indicating that the instrument 
measured dispositional proneness consistently over time. Over 56 studies were used to measure internal consistency of the TEOSQ instrument, resulting in Cronbach's alpha coefficients of $\alpha=.79$ and $\alpha=.81$ for the task and ego orientation scales respectively, representing an acceptable internal consistency. Validity of the TEOSQ was established by factorial validity, concurrent validity, and predictive validity. "Investigations employing exploratory factor analysis ... have continuously found support for the predominant two-dimensional structure of the TEOSQ" (Duda, 1998, p. 24). Duda (1998) administered the task and ego orientation sport scales and the Motivation Orientation Scales to examine the concurrent validity of the TEOSQ. The study resulted in an $r=.67$ and $r=.62$ respectively between the two scales, a strong positive correlation.

The QIRS perceived benefit scale measured the subjects' perception of the gains associated with their recreational sport involvement. The 22 item perceived benefit scale was modified to a 4- point Likert-type scale ranging from 1 (no benefit) to 4 (great benefit) for clarity and brevity within this tool. Reliability of the scale was implied by its psychometric properties (Forrester \& Beggs, 2005). Forrester and Beggs (2005) established construct validity of the perceived benefits scale through principal component analysis and confirmatory factor analysis. Results from the principal component analysis found the three subgroups: social, intellectual, and fitness, to account for 68.59 percent of the variance, while the confirmatory factor analysis indicated a good fit (RMSEA: .09; NFI: .98; CFI: .98). The structure of the scale, however, has been found to vary across empirical studies (Forrester \& Beggs, 2005; Lower, Turner, \& Petersen, 2013).

\section{Procedures}

An online survey tool (Qualtrics) was used to host the online survey instrument and collect questionnaire responses. The consent form and link to the online questionnaire was emailed to the sampling frame. To collect additional responses, the principal investigator attended recreational sport program classes, meetings, and competitions to administer the hard copy survey instrument. 
Following the completion of data collection, responses to the hard copy instrument were manually entered into Qualtrics to coalesce with the online responses.

\section{Statistical Analysis}

Statistical analysis of the data included: descriptive statistics, factor analyses, reliabilities, and structural equation modeling. The data was imported into SPSS Statistics 19 software to treat the data. The pattern of missing data was analyzed to determine the extent of missing data, for which multiple imputation with fully conditional specification was employed to treat the data to reduce the number of incomplete cases. Once missing data was treated, SPSS was used to produce a covariance matrix of the 12 achievement goal orientation variables which was then inputted to LISREL 9.0 software to conduct confirmatory factor analysis and test the theoretical dichotomous measurement model. To assess the factor loadings, the observed t-values of the paths in the model were compared against the critical value of $t$ for a two-tailed test at a $\alpha<.05$ level of significance. The global fit indices were evaluated once the statistical significance of the parameter estimates was determined. The fit of the measurement model was assessed through the following five indices: chi-square $\left(\chi^{2}\right)$, root-mean-square error of approximation (RMSEA), goodness-of-fit index (GFI), comparative fit index (CFI), and standardized root-mean square residual (SRMR). Criterion associated with an acceptable model fit, in regards to the five indices selected, include: a nonstatistically significant $\chi^{2}$, a RMSEA less than .100, a GFI and CFI greater than .90 or .95, and a SRMR less than .05 (Schumacker \& Lomax, 2010). Statistical significance was set at $\alpha<.05$ for all analyses, a commonly accepted probability level in the behavioral sciences (Ary, Jacobs, \& Sorensen, 2010). If the majority of fit indices indicated an acceptable model fit and there were no theoretically justifiable modification suggestions, the measurement model was considered to be theoretically and empirically supported. Following confirmatory factor analysis, reliability was calculated for each confirmed factor. For which constructs with a Cronbach's alpha of .70 or greater were 
considered to be reliable based upon the .70 standard set by Hair et al. (1998). Once the structure of the measurement model was confirmed and the reliability of the factors assessed, the data was reduced from 12 items to two factors by creating a mean task orientation variable and mean ego orientation variable based on the factor loadings.

Principal component analysis was conducted to examine the factor structure of the QIRS perceived benefit scale, for which any factor with an eigenvalue greater than 1.0 was retained (Stevens, 2009). Varimax and oblimin rotation were both employed to determine which rotation method was appropriate for the perceived benefit data based on the correlations among the factors. Each item's factor loading was examined for which any loading greater than .60 was deemed acceptable and maintained (Hair, Anderson, Tathan, \& Black, 1998); all items with factor loadings less than the .60 standard were removed. Any items with factor loadings that loaded similarly across multiple factors were assessed based on theory and past studies utilizing the scale to determine which factor to group the item with. Items found to load appropriately were grouped and then the reliability was calculated for each extracted factor, utilizing the .70 standard previously noted. Once the structure of the perceived benefit scale was determined, the data was reduced from 22 items to three factors by creating a mean social perceived benefits score, mean fitness perceived benefit score, and mean intellectual perceived benefit score based on the factor loadings.

A total quantitative recreational sport involvement variable was created by summing the subjects' recreational sport involvement in each of the three recreational sport program areas. In addition to performing data reduction techniques, normality was assessed through skewness and kurtosis of the created variables (i.e., task orientation, ego orientation, social perceived benefits, fitness perceived benefits, intellectual perceived benefits, and quantitative involvement). The normality assumption was considered to be met if the skewness and kurtosis statistics fell with the accepted range per Kendall and Stuart's (1958) standards (i.e., skewness: +2.00 to 2.00; kurtosis: +5.00 to -5.00$)$. 
Upon completing descriptive statistics, factor analyses, and reliabilities, SEM analyses were conducted, for which a polyserial correlation matrix was inputted into LISREL 8.8 software and the model relationships and parameters were specified. The observed tvalues for the individual paths in the mixture model were compared to the critical t-value previously noted to assess the model. Once statistical significance of the parameter estimates was examined, the fit of the proposed model was assessed through the global fit indices previously noted. Upon considering model modification suggestions, the asymptotic covariance matrix was added to generate robust statistics. The model was considered to be theoretically and empirically supported if there were no theoretically justifiable modification suggestions and the majority of fit indices indicated a good fitting model.

\section{Data}

\section{Results}

The sample consisted of a total of 1,881 recreational sport participants from a mid-sized institution. The pattern of missing data was analyzed in SPSS, which revealed that $37 \%(n=696)$ of the cases had missing data, accounting for approximately $12.63 \%$ of the total values. Multiple imputation with fully conditional specification was conducted to treat the data and reduce the number of cases with missing data. The multiple imputation resulted in 1,639 complete cases, transforming 454 of the incomplete cases. The number of eligible, complete cases was reduced from 1,639 to 1,564 based upon constraints placed on the total quantitative involvement variable. The involvement variable was constrained to greater than zero minutes and less than or equal to 1200 minutes for the purpose of this study. Involvement was fixed to be greater than zero minutes per week based upon the assertion that a subject must have contributed greater than zero minutes of sport involvement to experience benefits from that involvement. The variable was constrained to less than or equal to 1200 minutes (i.e., 20 hours) of recreational sport involvement per week as only .4\% of the sample reported greater than 1200 minutes of involvement, skewing the 
results, and student employment with the recreational sport department only allows for up to 20 hours of work per week. The resulting sample size used for all analyses comprised 1,564 cases.

\section{Sample}

The respondents consisted of $49 \%$ male $(n=763)$ and $45 \%$ female $(n=709)$, with 92 respondents missing gender data. The vast majority of respondents (88\%) ranged 18 to 22 years of age. The results indicated a representative academic class distribution with $18 \%$ freshmen respondents, $21 \%$ sophomore respondents, $21 \%$ junior respondents, $22 \%$ senior respondents, $8 \%$ graduate respondents, and 5\% non-student respondents, with 96 respondents missing data. The sample was predominately Caucasian (74\%); 8\% of respondents were Hispanic; 5\% African-American, 4\% Asian, 1\% Native American, and 2\% classified as 'other', with 100 respondents missing data.

\section{Confirmatory Factor Analysis}

The TEOSQ is designed to measure achievement goal orientations within the sport domain, with a dichotomous achievement goal framework. The dichotomous measurement model was tested through confirmatory factor analysis to confirm or disconfirm the factor loadings in order to inform data reduction. The 12 goal items (Table 1) were hypothesized to load onto two factors (i.e., task goal orientation, ego goal orientation) based upon the theoretical dichotomous achievement goal model. 
Table 1

Proposed factor loadings for dichotomous achievement goal

measurement model

\begin{tabular}{lll}
\hline $\begin{array}{l}\text { Observed } \\
\text { Variables }\end{array}$ & \multicolumn{1}{c}{ Variable Items } & \multicolumn{1}{c}{ Paths } \\
\hline Goal 1 & $\begin{array}{l}\text { I'm the only one who can do the play or skill } \\
\text { I learn a new skill and it makes me want to }\end{array}$ & Goal $1 \rightarrow$ Ego \\
Goal 2 & practice more & Goal $2 \rightarrow$ Task \\
Goal 3 & I can do better than my friends & Goal $3 \rightarrow$ Ego \\
Goal 4 & The others can't do as well as me & Goal $4 \rightarrow$ Ego \\
Goal 5 & I learn something that is fun to do & Goal $5 \rightarrow$ Task \\
Goal 6 & Others mess up and I don't & Goal $6 \rightarrow$ Ego \\
Goal 7 & I learn a new skill by trying hard & Goal $7 \rightarrow$ Task \\
Goal 8 & I score the most points/goals/hits, etc. & Goal $8 \rightarrow$ Ego \\
Goal 9 & Something I learn makes me want to go and & Goal $9 \rightarrow$ Task \\
Goal 10 & I'm the best & Goal $10 \rightarrow$ Ego \\
Goal 11 & A skill I learn really feels right & Goal $11 \rightarrow$ Task \\
Goal 12 & I do my very best & Goal $12 \rightarrow$ Task \\
\hline
\end{tabular}

Note. The following abbreviations were applied: Task: Task Orientation; Ego:

Ego Orientation.

A covariance matrix of the achievement goal orientation items was produced in SPSS and then inputted into LISREL 9.0 for confirmatory factor analysis. The largest factor loading for each factor was fixed to 1.0 and the factors were allowed to correlate. The objective of the analysis was to determine which factor loadings produced the best fitting model. The global fit indices for the dichotomous model $\left[\chi^{2}(53)=645, \mathrm{p}<.001\right.$; RMSEA: .085; GFI: .933; CFI: .951; SRMR: .056] suggest a good fitting model as the majority of the fit indices upheld the standards previously noted (i.e., RMSEA $<.100$, GFI $>.90$, CFI $>.95$ ). Confirmatory factor analysis found all factor loadings significant at the $\alpha<.05$ level of significance. The largest standardized residuals were associated with observed variables which loaded onto the same factor; the residuals 
did not suggest a modification involving the factor loadings. The MI and EPC statistics were examined, for which Goal 11 was suggested to load onto the ego factor rather than the task factor. However, the $\mathrm{R}^{2}$ for Goal 11(.419) suggests that the observed variable explains a moderate amount of the variance in task orientation. The other modification suggestions were either not theoretically justifiable or focused on adding error covariances (which was not the objective of the confirmatory factor analysis).

The dichotomous measurement model was modified to incorporate the path from Goal 11 to ego orientation to examine if the fit of the model improved. The modification was found to deteriorate the fit of the model $\left[\chi^{2}(53)=1233.15, p<.001\right.$; RMSEA: .119; GFI: .890; CFI: .912; SRMR: .118]. Therefore, the modification was not maintained and the proposed dichotomous measurement model (Figure 2) was upheld. 
Figure 2

Path diagram for proposed dichotomous achievement goal measurement model with standardized solutions

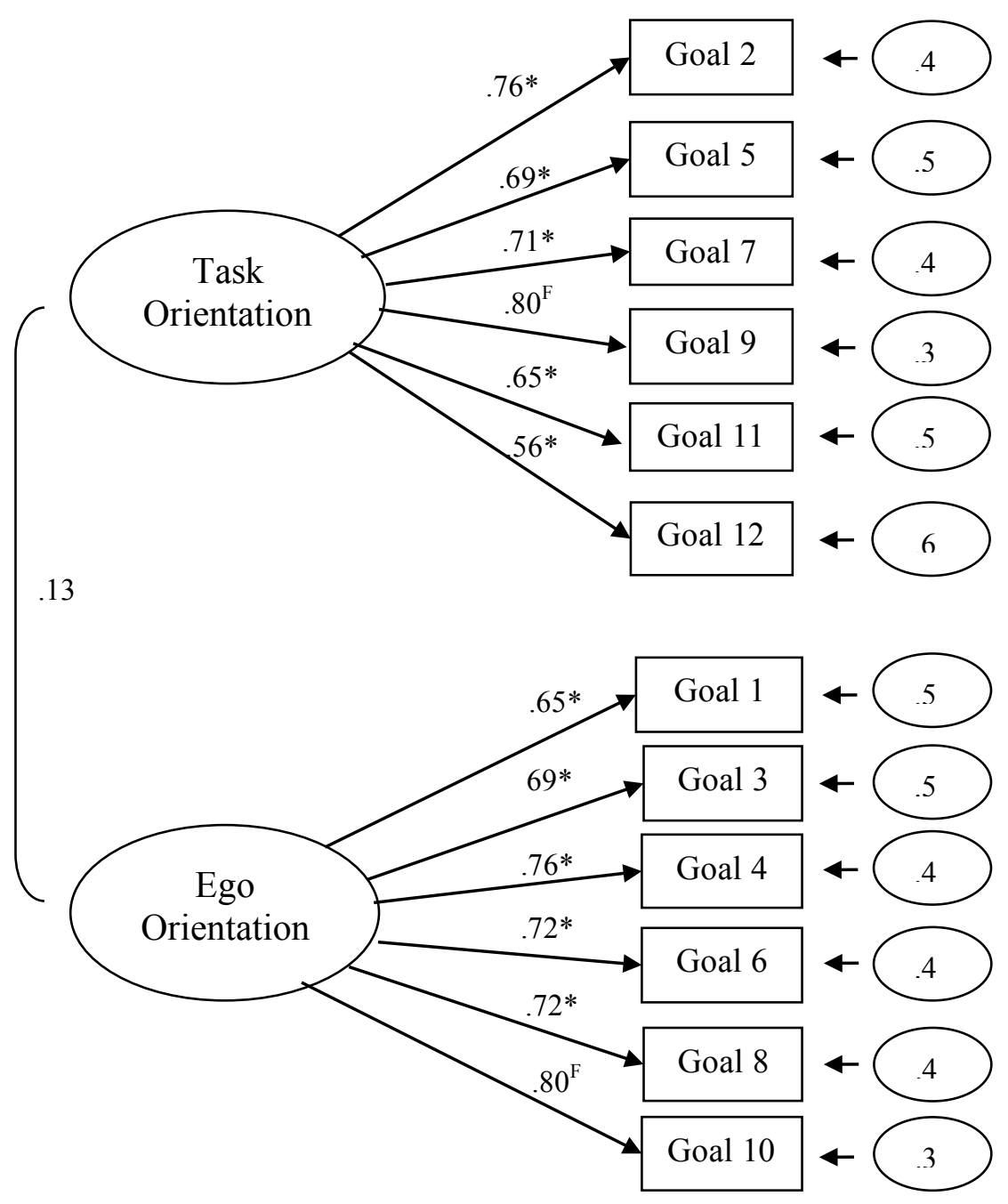

Note. The fixed factors are denoted by 'F'. ${ }^{*} p<.001$. 
Once the factor structure of the dichotomous achievement goal model was confirmed, reliability statistics were conducted for each factor. Cronbach's alpha coefficients for both factors (task $\alpha=$ .849; ego $\alpha=.869$ ) suggested high reliability based on Hair et al.'s (1998) .70 standard previously noted. The data was then reduced from 12 goal items to two goal orientation measures of central tendency. A mean task goal orientation and mean ego goal orientation was calculated for each subject based on the confirmed factors and factor loadings.

\section{Principal Component Analysis}

The QIRS perceived benefit scale was designed to measure perceived benefits of recreational sport involvement, with a three factor structure. The perceived benefit items have not loaded consistently in past empirical studies, therefore principal component analysis was conducted to explore the factor structure of the scale. Principal component analysis was run with both a varimax rotation and oblimin rotation to determine which rotation method was most appropriate. The correlations found among the factors ranged from .311 to .441 suggesting non-orthogonal factors; therefore oblimin rotation was selected for the analysis. Results of the analysis can be viewed in Table 2. The three extracted factors (based on an eigenvalue greater than 1.0) were found to account for $57.3 \%$ of the variance cumulatively, for which the first component (i.e., social perceived benefits) accounted for $41.1 \%$. The 'sports skills' perceived benefit item did not meet the .60 factor loading standard previously noted; as a result the item was eliminated from future analyses. The 'defining problems' and 'problem-solving skills' perceived benefit items loaded similarly onto multiple components, requiring theoretical justification for the factor loadings. Both items have loaded onto the intellectual factor in previous empirical research and therefore were selected to load onto the intellectual factor for the subsequent analyses of the current study. 
Table 2

Principal component analysis with oblimin rotation for perceived benefit items

\begin{tabular}{|c|c|c|c|}
\hline \multirow[b]{2}{*}{ Perceived Benefit Items } & \multicolumn{3}{|c|}{ Component } \\
\hline & Social & Fitness & Intellectual \\
\hline Sense of adventure & 0.601 & 0.459 & 0.391 \\
\hline Group cooperation skills & 0.797 & 0.325 & 0.305 \\
\hline Respect for others & 0.750 & 0.401 & 0.378 \\
\hline Communication skills & 0.795 & 0.294 & 0.406 \\
\hline Belonging/association & 0.751 & 0.464 & 0.254 \\
\hline Leadership skills & 0.757 & 0.303 & 0.456 \\
\hline Developing friendships & 0.697 & 0.401 & 0.255 \\
\hline Sports skills & $0.579 *$ & 0.536 & 0.310 \\
\hline Improved self-confidence & 0.531 & 0.662 & 0.350 \\
\hline Feeling of physical well-being & 0.442 & 0.760 & 0.125 \\
\hline Sense of accomplishment & 0.523 & 0.702 & 0.199 \\
\hline Weight control & 0.252 & 0.722 & 0.401 \\
\hline Fitness & 0.324 & 0.794 & 0.160 \\
\hline Physical strength & 0.329 & 0.768 & 0.347 \\
\hline Stress reduction & 0.272 & 0.653 & 0.236 \\
\hline Balance/coordination & 0.413 & 0.707 & 0.390 \\
\hline Defining problems & 0.654 & 0.277 & 0.690 \\
\hline Problem-solving skills & 0.703 & 0.287 & 0.659 \\
\hline Study habits & 0.300 & 0.359 & 0.756 \\
\hline Time-management skills & 0.456 & 0.507 & 0.666 \\
\hline Understanding written information & 0.442 & 0.283 & 0.830 \\
\hline Handling several tasks at once & 0.560 & 0.397 & 0.693 \\
\hline
\end{tabular}

Note. Factor loadings $>.60$ are in boldface. ${ }^{*}$ Item fell below the .60 threshold and was removed. 
Reliability analyses were conducted for the three extracted factors, for which Cronbach's alphas suggested high reliability for all factors (social $\alpha=.869$; fitness $\alpha=.872$; intellectual $\alpha=.857$ ) based on the .70 standard previously noted. The data was then reduced from 22 perceived benefit items to three measures of perceived benefits based on central tendency. A mean social perceived benefit, fitness perceived benefit, and intellectual perceived benefit score was calculated for each subject based on the extracted factors and factor loadings.

\section{Normality}

Normality is a necessary assumption for SEM analysis as nonnormal data may affect results such as parameter estimates, standard errors, and fit indices (Schumacker \& Lomax, 2010). The normality assumption was assessed through skewness and kurtosis statistics of the observed variables. The skewness and kurtosis statistics for the goal orientation variables and perceived benefit variables were found to fall within the accepted ranges previously noted (Kendall \& Stuart, 1958). The continuous quantitative involvement variable was found to be positively skewed and platykurtic (skewness: 2.685; kurtosis: 8.482).

Involvement. To address the normality violation of the continuous quantitative involvement variable, the researcher created an ordinal variable which divided the sample into 20 categories based on level of involvement (i.e., range of minutes per week for which each category constituted consecutive ranges of one hour; e.g., $1=1-60 \mathrm{~min}$., $2=61-120 \mathrm{~min}, . .$.$) . The ordinal quantitative$ involvement variable was also found to be nonnormal. SEM analyses were conducted for both types of quantitative involvement variables (i.e., continuous and ordinal), for which the continuous variable was found to cause multicollinearity issues. Therefore, the ordinal quantitative involvement variable was maintained. Descriptive statistics of the observed variables can be found in Table 3. 
Table 3

Descriptive statistics of observed variables

\begin{tabular}{lccccc}
\hline Variables & $n$ & $M$ & $S D$ & Skewness & Kurtosis \\
\hline $\begin{array}{l}\text { Ordinal Quantitative } \\
\text { Involvement }\end{array}$ & 1564 & 2.892 & 3.112 & 2.719 & 8.630 \\
Task Orientation & 1564 & 4.001 & 0.672 & -1.468 & 4.157 \\
$\begin{array}{l}\text { Ego Orientation } \\
\text { Social Perceived }\end{array}$ & 1564 & 2.788 & 0.905 & 0.197 & -0.421 \\
$\begin{array}{l}\text { Benefits } \\
\text { Fitness Perceived }\end{array}$ & 1564 & 3.048 & 0.652 & -0.434 & -0.154 \\
$\begin{array}{l}\text { Benefits } \\
\text { Intellectual Perceived }\end{array}$ & 1564 & 3.140 & 0.603 & -0.572 & 0.030 \\
Benefits & 1564 & 2.536 & 0.746 & 0.094 & -0.501 \\
\hline
\end{tabular}

\section{Model}

The structural model proposed (Figure 1) hypothesizes achievement goal orientations to predict quantitative sport involvement which in turn predicts perceived benefits of sport involvement. The achievement goal variables were not hypothesized to measure a latent variable as empirical research has found inconsistent results regarding the consequences of a task and ego goal orientation; as such the unique relationships between the individual goal orientations and quantitative involvement were of interest. The quantitative involvement ordinal variable was hypothesized to measure an involvement latent variable based upon Astin's (1999) conceptualization of involvement, which measures involvement both quantitatively and qualitatively. The latent variable suggests a greater involvement construct, supporting Astin's conceptualization. The perceived benefit variables were hypothesized to measure a perceived benefit latent variable as the observed variables were extracted components of an overall perceived benefit measure.

The proposed structural model contained both interval and ordinal observed variables; therefore the model was treated as a mixture model. The observed variables were defined as either 
continuous or ordinal in PRELIS 9.0 and then a polyserial correlation matrix and asymptotic covariance matrix of the observed variables was produced and saved for the SEM analyses. The polyserial correlation matrix can be found in Table 4 . The first run included the polyserial matrix only; the means and standard deviations were specified as a correlation matrix was used to account for the relationships among the observed variables. The observed and latent variables and the relationships among those variables were specified, with the achievement goal variables correlating. As a single observed variable (i.e., quantitative involvement) was used to assess the involvement latent variable, it is assumed that the latent variable is perfectly measured by the single observed variable; therefore the factor loading was fixed to 1.0 and the measurement error was fixed to 0.0 . Of the perceived benefit observed variables, the social perceived benefit factor loading was fixed to 1.0 based upon the results of the principal component analysis.

Table 4

Polyserial correlation matrix of observed variables

\begin{tabular}{lcccccc}
\hline Observed Variables & 1 & 2 & 3 & 4 & 5 & 6 \\
\hline 1. Quantitative Involvement & 1.000 & & & & & \\
2. Task Goal Orientation & 0.097 & 1.000 & & & & \\
3. Ego Goal Orientation & 0.091 & 0.123 & 1.000 & & & \\
4. Social Perceived Benefits & 0.075 & 0.102 & -0.049 & 1.000 & & \\
5. Fitness Perceived Benefits & 0.136 & 0.232 & 0.022 & 0.574 & 1.000 & \\
6. Intellectual Perceived & & & & & & \\
$\quad$ Benefits & 0.038 & 0.005 & 0.010 & 0.698 & 0.541 & 1.000 \\
\hline
\end{tabular}

Note. Quantitative Involvement refers to ordinal variable.

The model was over-identified with 21 unique values in the covariance matrix $\mathrm{S}$ (based on six observed variables) and 13 free parameters, indicating room for model modification. The generalized least squares (GLS) and maximum likelihood (ML) methods of estimation were employed to determine the most appropriate method 
of estimation for the model. The ML method is recommended for slight to moderate nonnormal interval and ordinal data, while the GLS method is recommended for severely nonnormal interval and ordinal data (Schumacker \& Lomax, 2010). The ML method of estimation was found to produce a better fitting model and therefore was used for all SEM analyses.

The mixture model, using a polyserial correlation matrix, was run, for which all factor loadings and structural coefficients were found significant at the $\alpha<.05$ level of significance. The global fit indices of the proposed model (Table 5) suggest a poor fitting model as three of the five indices do not meet the standards previously noted (i.e., non-significant $\chi^{2}$; RMSEA < .100; SRMR $<.05$ ). The $\mathrm{R}^{2}$ values were moderately strong for the measurement equations but small for the structural equations. When considering the modification suggestions, the only theoretically justifiable suggestion was adding a path between the task orientation observed variable and perceived benefits latent variable (anticipated decrease in chi-square: 14.7).

Table 5

Structural equation modeling goodness of fit statistics for the structural model

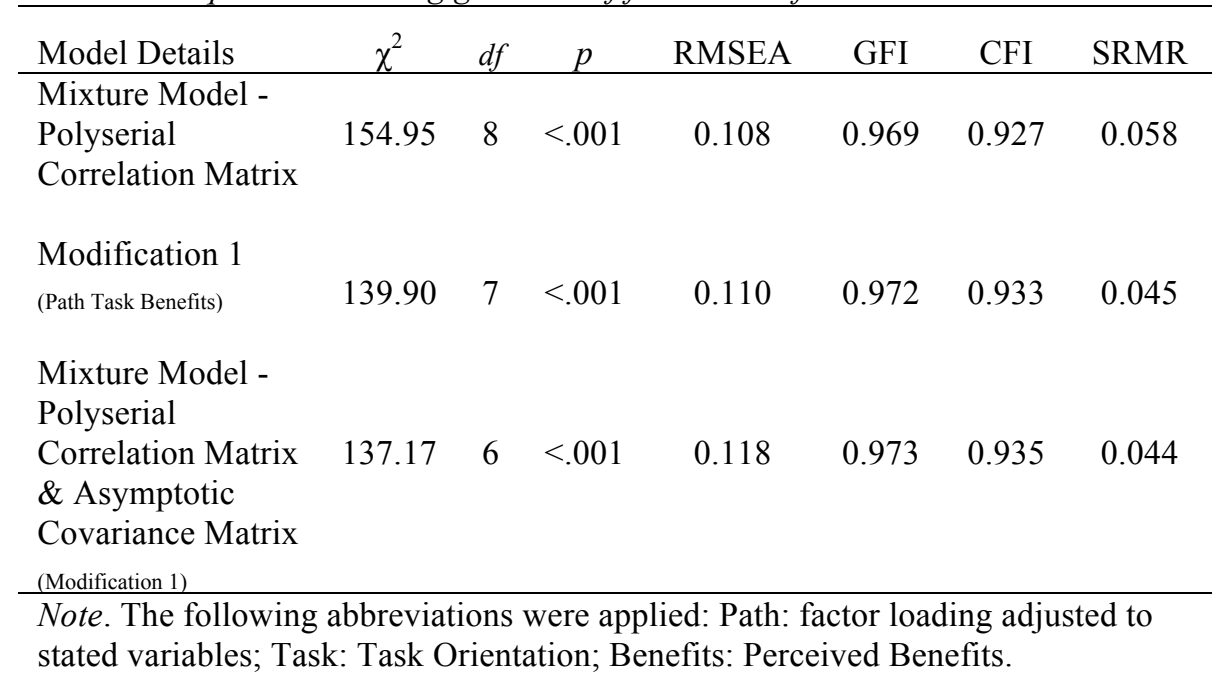


The mixture model was modified by adding a path from the task orientation observed variable to the perceived benefits latent variable to determine if the fit of the model would improve. All factor loadings and structural coefficients remained significant at the $\alpha<.05$ level of significance. The global fit indices for the modified mixture model (Table 5) suggest a good fitting model as the majority of indices upheld the standards previously noted (i.e., GFI $>.95$, CFI $>.90, \mathrm{SRMR}<.05)$. The structural equation $\mathrm{R}^{2}$ statistics also improved, supporting the modification to the model. The modification indices were reviewed for which no suggestions were found theoretically justifiable. Thus the modified model (Figure 3), with three indices indicating a good fit, was found to be the best fitting model.

As the structural model is considered a mixture model with both continuous and ordinal data, the asymptotic covariance matrix was inputted to provide additional information for the SEM analysis and generate robust statistics. The final modified model was rerun using both the polyserial correlation matrix and asymptotic covariance matrix. The global fit indices improved slightly (Table 5), supporting the inclusion of the asymptotic covariance matrix.

The proposed structural model was partially supported in that all relationships hypothesized were found statistically significant at the $\alpha<.05$ level of significance. The mixture model was modified by adding a path between the task orientation observed variable and perceived benefits latent variable to improve the fit of the model, indicating that the proposed model did not provide the best fit. 
Figure 3

Path diagram of modified structural model with standardized solutions

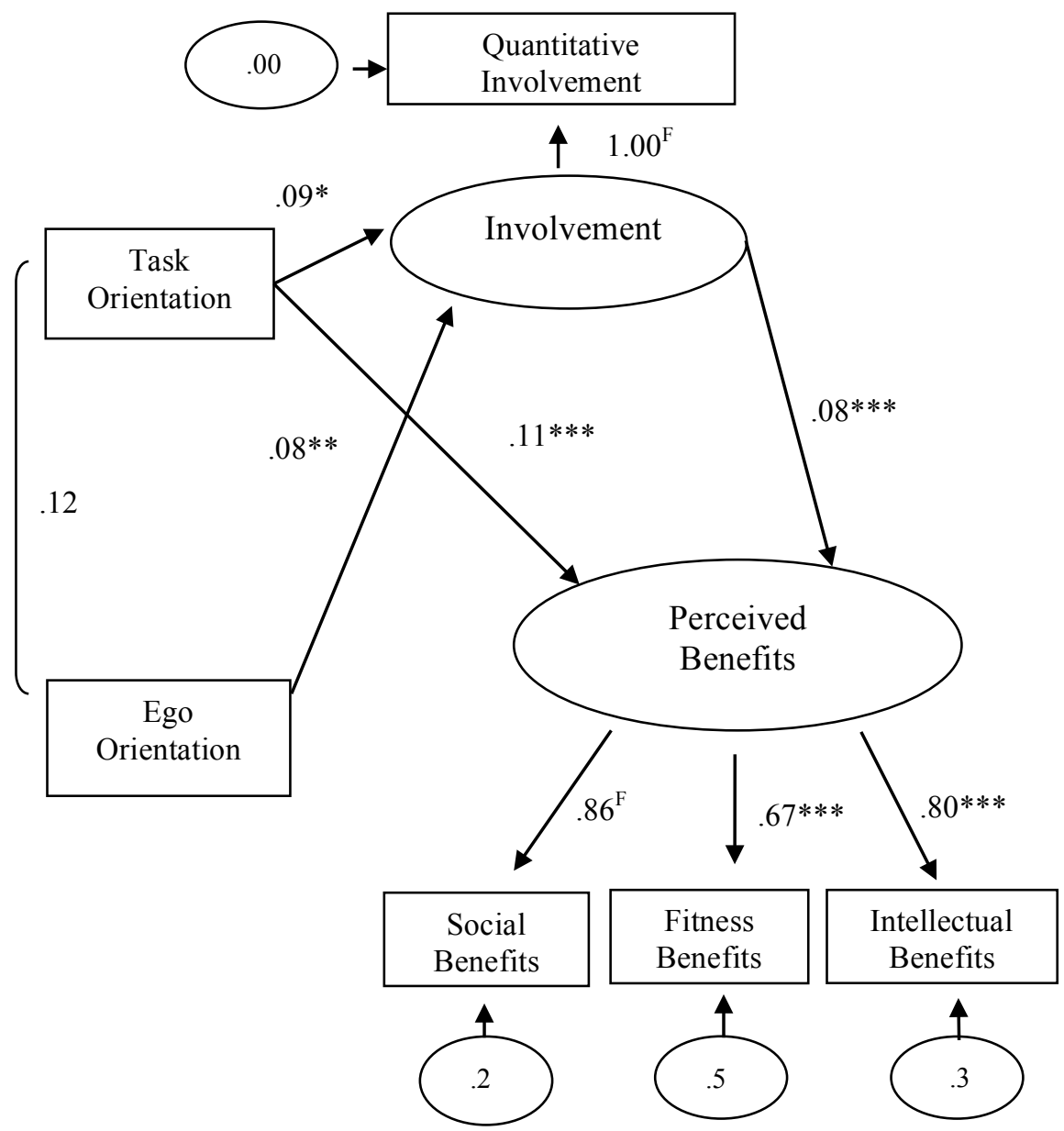

Note: The fixed factors are denoted by ' $F$ '. ${ }^{* *} p<.05$, ${ }^{* *} p<.001$.

\section{Discussion}

A mixture model was proposed and tested, examining the relationships among achievement goal orientations (i.e., task and ego), recreational sport involvement (i.e., quantitative involvement), and perceived benefits of recreational sport involvement (i.e., social, fitness, and intellectual benefits). Several procedures were conducted 
prior to testing the model, including confirmatory factor analysis, principal component analysis, and descriptive statistics to examine the assumption of normality. Confirmatory factor analysis was conducted to test the structure of the TEOSQ scale, which utilizes the dichotomous achievement goal model. The dichotomous measurement model was upheld, confirming the proposed factor structure of the TEOSQ. The confirmatory factor analysis and reliability analyses of the TEOSQ suggest it to be an empirically sound instrument for testing the dichotomous achievement goal model within the sport domain.

Principal component factor analysis was conducted to examine the structure of the QIRS perceived benefit scale. The factor loadings of the perceived benefit scale have varied across empirical studies, which is why an exploratory factor analysis technique was employed for the current study. The three factor structure of the scale was upheld, supporting past research which has extracted three components. An oblimin rotation was found to be most appropriate based upon the correlations found among the perceived benefit factors. As all items were developed to measure a comprehensive construct (perceived benefits of recreational sport), the correlation among the types of perceived benefits is justifiable. The constructs reflecting the extracted components proposed in past studies (i.e., social, fitness, and intellectual perceived benefits) were suitable for the extracted components of the current study and therefore were maintained. Of the 22 perceived benefit items, the 'sport skills' item had a factor loading less than the .60 standard previously noted. Of the extracted components, the 'sport skills' item was anticipated to load onto the fitness component. The low factor loading may have been influenced by the population investigated, which included group fitness participants who may not relate to the perceived benefit item, which has an emphasis on sport. Ultimately, the confirmatory factor and principal component analyses were used as data reduction techniques to reduce the TEOSQ from 12 items to two components and the QIRS perceived benefit scale from 22 items to three components. The data was 
reduced for the purpose of proposing a more parsimonious structural model.

When assessing the normality of the observed variables, the quantitative involvement variable (both continuous and ordinal) was found to be nonnormal. The distribution of the data indicates that approximately $77 \%$ of subjects reported involvement less than or equal to 180 minutes per week, while the involvement variable ranged from one to 1200 minutes. The distribution of the data is likely due to the structure of the intramural sport program and inclusion of recreational sport student employees in the study. The intramural sport program at the institution studied schedules only two sports concurrently, which limits participation to approximately 120 minutes per week and may account for the positively skewed data. The responses indicating close to 1200 minutes of involvement may be explained by the inclusion of recreational sport student employees in the study as many student employees are also involved in recreational sport programs as participants and may consider recreational sport involvement to include both work and voluntary time. Although both involvement variables (i.e., continuous and ordinal) were found to be nonnormal, the ordinal variable was selected for analyses to avoid the multicollinearity issues that arose with the continuous variable.

With the inclusion of continuous and ordinal variables, a mixture model was used for SEM analysis. Based upon the nonnormality of the quantitative involvement variable, both the ML and GLS methods of estimation were employed to determine the most appropriate estimation method for the data. The maximum likelihood method was found to produce a better fitting model, suggesting that the violation of normality was minimal. The violation was likely not a major issue as only one of the six observed variables were found to be nonnormal.

The chi-square global fit indice was found nonsignificant for all SEM analyses, suggesting poor fitting models. However, the chisquare model-fit criterion is sensitive to sample size (Schumacker \& Lomax, 2010) and therefore was likely influenced by the study's large sample $(n=1564)$. The global fit indices for the final modified 
mixture model suggested a good fitting model as the majority of fit indices met the standards previously noted. Inclusion of the asymptotic covariance matrix within the analysis improved the fit of the mixture model, supporting the assertion that its inclusion yields robust statistics within mixture models.

Although the model was found to have a good fit, the structural coefficients and squared multiple correlations for the structural equations were relatively small suggesting moderate to weak predictive relationships among the achievement goal orientation, quantitative involvement, and perceived benefits of involvement variables. The positive predictive relationship between task orientation and the involvement and perceived benefits factors supports past research which has consistently found a task orientation associated with positive achievement-related outcomes. The positive predictive relationship between ego orientation and the involvement factor provides additional insight into the outcomes of an ego orientation as there have been inconsistent findings in past studies. Lastly, the positive predictive relationship between the involvement and perceived benefits of involvement factors supports Astin's (1999) assertion of a direct positive relationship between involvement and student development. In contrast to the structural coefficients, the perceived benefit factor loadings were relatively large, supporting the use of the three components (i.e., social, fitness, and intellectual perceived benefits) as a measure of perceived benefits of recreational sport involvement.

\section{Conclusions}

Limited research has investigated the predictive relationship between achievement goal orientations, sport involvement, and perceived benefits of sport participation within a structural model. Examining how goal orientation, sport involvement, and program perceived benefits interact can help sport practitioners better understand retention, participant experiential differences, programmatic weaknesses and strengths, the optimal program structure, as well as other significant components of a successful program. 
This study was designed to examine the premise that achievement goals predict achievement-related behaviors and outcomes. Results from this study support the supposition that a task orientation should be enhanced and proposal that an ego orientation need not be depressed but can actually result in positive achievement-related outcomes. Sport program facilitators can influence participant goal orientations by consistently emphasizing the desired achievement goal through purposeful behaviors. Behaviors that project a task goal orientation emphasize personal improvement, effort, and learning. Sport instructors can set goals focusing on skill development to motivate and evaluate progress, present skill modifications and alternatives, offer instruction and constructive feedback in private, and reinforce effort through encouragement and feedback. Competition is an inherent element of sport, emphasizing performance, winning, and rewards characteristics often associated with an ego orientation. Based upon the current study, an ego orientation need not be discouraged. Future studies should seek to confirm or disconfirm this finding as there has been inconsistent results regarding the achievement-related outcomes of an ego orientation. The results from this study have several implications for sport practitioners regarding the outcomes of program participation and the factors influencing those outcomes. Generalizability of the results is limited due to the single institution research model. Therefore, further research should be conducted to support the findings of this study or offer additional explanations for these findings.

\section{Limitations and Recommendations}

This study offers many opportunities for expansion to better understand the interaction of goal orientation, program involvement, and perceived benefits associated with program participation. The constructs selected to represent achievement-related behaviors and outcomes can be developed further to provide a more comprehensive outlook of the consequences of achievement goal orientations. The involvement measure within the current study was limited to quantitative involvement; future research should consider including 
qualitative involvement (e.g., degree of effort expenditure) for additional insight to the overarching involvement factor. The quantitative involvement variable was found to be nonnormal, which has the potential to affect the results of SEM analysis. Future studies investigating quantitative involvement may consider using a scale rather than an open-ended question, to address the normality issue. Perceived benefits of program participation were selected to reflect achievement-related outcomes; however, the measure used in this study focused only on positive achievement-related outcomes. Future studies should consider incorporating both positive and negative outcomes for a more comprehensive analysis.

Since the conception of the dichotomous achievement goal model, the achievement goal theory has expanded to incorporate the valence dimension (i.e., approach-avoidance motivation) and divide the competency dimension into three elements based on standards of evaluation (task-based, self-based, other-based). The trichotomous, $2 \times 2$, and $3 \times 2$ achievement goal models add complexity and potentially allow for more in-depth analysis. Expanding this study to incorporate the additional factors may offer more in-depth insight to the interaction between the variables. Currently, only the dichotomous and $2 \times 2$ frameworks have published measures developed for the sport domain. Researchers should consider examining the trichotomous and $3 \times 2$ models within the sport domain in order to determine which approach is most appropriate within the sport context.

The model focused on consequences of achievement goal orientations and can be expanded to integrate antecedents of achievement goal orientations in order to better understand the construct and its interaction with other achievement-related variables. The terms consequences and outcomes were not indented to indicate any causality among the variables, merely predictive relationships with a logical progression (i.e., goal orientation $\rightarrow$ involvement $\rightarrow$ perceived benefits). Longitudinal research with an experimental design may be considered if investigation of causality among the achievement-related constructs is of interest. 


\section{References}

Adie, J. W., Duda, J. L., \& Ntoumanis, N. (2008). Achievement goals, competition appraisals, and the psychological and emotional welfare of sport participants. Journal of Sport \& Exercise Psychology, 30, 302-322.

Ames, C. (1992). Classrooms, goals, structures, and student motivation. Journal of Educational Psychology, 84, 261-271.

Ames, C., \& Archer, J. (1988). Achievement goals in the classroom: Students' learning strategies and motivation processes. Journal of Educational Psychology, 80 (3), 260-267.

Artinger, L., Clapham, L., Hunt, C., Meigs, M., Milord, N., Sampson, B., \& Forrester, S. A. (2006). The social benefits of intramural sports. NASPA Journal, 43 (1), 69-86. Ary, D., Jacobs, L. C., \& Sorensen, C. (2010). Introduction to research in education (8th ed.). Belmont, CA: Wadsworth.

Astin, A. W. (1999). Involvement in learning revisited: Lessons we have learned. Journal of College Student Development, 40 (5), 587-598.

Astin, A. W. (1999). Student involvement: A developmental theory for higher education. Journal of College Student Development, 40 (5), 518-529.

Biddle, S. J., Want, C. K., Kavussanu, M., \& Spray, C. M. (2003). Correlates of achievement goal orientations in physical activity: A systematic review of research. European Journal of Sport Science, 3 (5), 1-20.

Bourgeois, A., LeUnes, A., Burkett, S., Dragges-Bourgeois, T., Friend, J., \& Meyers, M. (1995, Spring). Factors influencing intramural sport participation. NIRSA Journal, 44-48.

Cooney, L. (1979). Sports clubs: Their place within the total intramural-recreational sports program. Journal of Physical Education and Recreation, 50 (3), 40-41.

Dishman, R. K., \& Chambliss, H. O. (2010). Exercise psychology. In J. M. Williams ( $6^{\text {th }}$ Ed.), Applied sport psychology: Personal growth to peak performance (p.497-518). Boston: McGraw-Hill. 
Duda, J. L. (1989). Relationship between task and ego orientation and the perceived purpose of sport among high school athletes. Journal of Sport \& Exercise Psychology, 11 (3), 318-335.

Duda, J. L. (1998). Advances in sport and exercise psychology measurement. Morgantown: Fitness Information Technology.

Duda, J. L. (2005). Motivation in sport: The relevance of competence and achievement goals. In Elliot, A.J., \& Dweck, C.S. (Eds.), Handbook of competence and motivation (pp. 318-335). New York: Guilford Press.

Duda, J. L., \& Whitehead, J. (1998). Measurement of goal perspectives in the physical domain. In J. Duda (Ed.), Advances in sport and exercise psychology measurement (pp. 21-48). Morgantown, WV: Fitness Information Technology.

Elliot, A. J. (1999). Approach and avoidance motivation and achievement goals. Educational Psychologist, 34 (3), 169189.

Elliot, A. J. (2005). A conceptual history of the achievement goal construct. In A. J. Elliot \& C. S. Dweck (Eds.), Handbook of competence and motivation (pp. 52-72). New York, NY: The Guilford Press.

Elliot, A. J., \& Covington, M. V. (2001). Approach and avoidance motivation. Educational Psychology Review, 13 (2), 73-92.

Elliot, A. J., \& Harackiewicz, J. M. (1996). Approach and avoidance achievement goals and intrinsic motivation: A meditational analysis. Journal of Personality and Social Psychology, 70 (3), 461-475.

Elliot, A. J., \& McGregor, H. A. (2001). A 2 x 2 achievement goal framework. Journal of Personality and Social Psychology, 80 (3), 501-519.

Elliot, A. J., Murayama, K., \& Pekrun, R. (2011). A 3 x 2 achievement goal model. Journal of Educational Psychology, 103 (3), 632-648. 
Forrester, S. A., \& Beggs, B. (2005). Validation of the quality and importance of recreational sports scale. [Abstract] Canadian Congress on Leisure Research accessed online at http://lin.ca/Uploads/cclr11/CCLR11-35.pdf

Haines, D. J., \& Fortman, T. (2008). The college recreational sports learning environment. Recreational Sports Journal, 32, 5261.

Hair, J. F., Anderson, R. E., Tatham, R. L., Black, W. C. (1998). Multivariate data analysis with readings. Englewood, NJ: Prentice Hall.

Hall, D. A. (2006). Participation in a campus recreation program and its effect on student retention. Recreational Sports Journal, 30 (1), 40-45.

Hendricks, J. W., \& Payne, S. C. (2007). Beyond the big five: Leader goal orientation as a predictor of leadership effectiveness. Human Performance, 20 (4), 317-343.

Kendall, M. G., \& Stuart, A. (1958). The advanced theory of statistics. New York, NY: Hafner.

Kuh, G. D. (1991). Involving colleges: Successful approaches to fostering student learning and development outside the classroom. San Francisco: Jossey-Bass.

Lang, P. J. (1995). The emotion probe: Studies of motivation and attention. American Psychologist, 50 (5), 372-285.

LeUnes, A. (2008). Sport psychology ( $4^{\text {th }}$ ed.). New York, NY: Psychology Press.

Lewin, K., Dembo, T., Festinger, L., \& Sears, P. S. (1944). Level of aspiration. In J. Hunt (Ed.), Personality and the behavior disorders (pp. 333-378). New York, NY: Ronald Press.

Lindsey, R., \& Sessoms, E. (2006). Assessment of a campus recreation program on student recruitment, retention, and frequency of participation across certain demographic variables. Recreational Sports Journal, 30 (1), 30-39.

Lower, L. M., Turner, B. A., \& Petersen, J. C. (2013). A comparative analysis of perceived benefits of participation between recreational sport programs. Recreational Sports Journal, 37, 66-83. 
Moller, A. C., \& Elliot, A. J. (2006). The 2x2 achievement goal framework: An overview of empirical research. In A. Mitel (Ed.), Focus on educational psychology (pp. 307-326). New York, NY: Nova Science Publishers, Inc.

National Intramural-Recreational Sports Association (NIRSA). (2000). Quality and importance of recreational services (QIRS). Champaign, IL: Human Kinetics.

National Intramural-Recreational Sports Association. (2004). The value of recreational sports in higher education. Corvallis, Oregon: NIRSA.

Nicholls, J. G. (1984). Achievement motivation: Conceptions of ability, subjective experience, task choice, and performance. Psychological Review, 91 (3), 328-346.

Nicholls, J. G. (1989). The competitive ethos and democratic education. Cambridge, MA: Harvard University Press.

NIRSA/NASPA Consortium. (2010). 2010 NIRSA/NASPA Consortium campus recreation impact study. Retrieved July 12, 2012, from http://www.nirsa.org/AM/Template.cfm?Section=Files\&Tem plate $=/$ MembersOnly.cfm \&ContentFileID $=11377$

Roberts, G. C. (2001). Understanding the dynamics of motivation in physical activity. In G. Roberts (Ed.), Advances in motivation in sport and exercise (pp. 1-50). Champaign, IL: Human Kinetics.

Roberts, G. C., Treasure, D. C., \& Balague, G. (1998). Achievement goals in sport: The development and validation of the perception of success questionnaire. Journal of Sports Sciences, 16, 337-347.

Roberts, G. C., Treasure, D. C., \& Conroy, D. E. (2007). Understanding the dynamics of motivation in sport and physical activity: An achievement goal interpretation. In G. Tenenbaum, \& R. Eklund (Eds.), Handbook of sport psychology ( $3^{\text {rd }}$ ed.) (pp. 3-30). Hoboken, NJ: John Wiley \& Sons, Inc. 
Roberts, G. C., Treasure, D. C., \& Kavussanu, M. (1996).

Orthogonality of achievement goals and its relationship to beliefs about success and satisfaction in sport. The Sport Psychologist, 10, 398-408.

Sage, L. D., \& Kavussanu, M. (2008). Goal orientations, motivational climate, and prosocial and antisocial behaviour in youth football: Exploring their temporal stability and reciprocal relationships. Journal of Sports Sciences, 26 (7), 717-732.

Schumacker, R. E. \& Lomax, R. G. (2010). A beginner's guide to structural equation modeling ( ${ }^{\text {rd }}$ ed.). New York:

Routledge.

Staurowsky, E. J., \& Abney, R. (2011). Intercollegiate athletics. In P. Pedersen, J. Parks, J. Quarterman, \& L. Thibault (Eds.), Contemporary sport management (pp. 142-163). Champaign, IL: Human Kinetics.

Stevens, J. P. (2009). Applied multivariate statistics for the social sciences $\left(5^{\text {th }}\right.$ ed. $)$. New York: Routledge.

The Ohio State University. (2003, April). 2002 QIRS - general report. Retrieved from http://studentlife.osu.edu/pdfs/assess_recreation_report.pdf 